\title{
CAEP Acute Atrial Fibrillation/Flutter Best Practices Checklist
}

Ian G. Stiell, MD, MSc ${ }^{* \dagger}$; Frank X. Scheuermeyer, MD, MHSc ${ }^{\ddagger}$; Alain Vadeboncoeur, MD ${ }^{\S \pi}$; Paul Angaran, MD; Debra Eagles, MD, $\mathrm{MSc}^{*{ }^{\dagger}}$; Ian D. Graham, $\mathrm{PhD}^{\dagger * *}$; Clare L. Atzema, MD, MSc ${ }^{\dagger+\neq}$; Patrick M. Archambault, MD, MSc ${ }^{\S \S}$; Troy Tebbenham, MD ${ }^{\text {Iा; }}$; Kerstin de Wit, MD, MSc ${ }^{\text {III. }}$ Andrew D. McRae, MD, PhD***; Warren J. Cheung, MD, MMEd ${ }^{*{ }^{*}}$; Marc W. Deyell, MD, MSc ${ }^{{ }^{+\dagger}}$; Geneviève Baril,

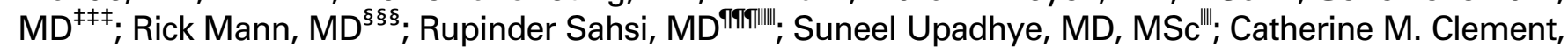
$\mathrm{RN}^{\dagger}$; Jennifer Brinkhurst, $\mathrm{BAH}^{\dagger}$; Christian Chabot ${ }^{* * *}$; David Gibbons ${ }^{\dagger++\dagger}$; Allan Skanes, $\mathrm{MD}^{\ddagger \neq \neq \neq}$

For a French translation of this position statement, please see the Supplementary Material at DOI: 10.107/cem.2018.26

The CAEP Acute Atrial Fibrillation/Flutter Best Practices Checklist was created to assist emergency physicians in Canada and elsewhere manage patients who present to the emergency department (ED) with acute/recentonset atrial fibrillation or flutter. The checklist focuses on symptomatic patients with acute atrial fibrillation (AAF) or flutter (AAFL), i.e. those with recent-onset episodes (either first detected, recurrent paroxysmal or recurrent persistent episodes) where the onset is generally less than 48 hours but may be as much as seven days. These are the most common acute arrhythmia cases requiring care in the ED. ${ }^{1,2}$ Canadian emergency physicians are known for publishing widely on this topic and for managing these patients quickly and efficiently in the ED. ${ }^{3-5}$

This project was funded by a research grant from the Canadian Arrhythmia Network and the resultant guidelines have been formally recommended by the
Canadian Association of Emergency Physicians (CAEP). We chose to adapt, for use by emergency physicians, existing high-quality clinical practice guidelines (CPG) previously developed by the Canadian Cardiovascular Society (CCS).$^{6-8}$ These CPGs were developed and revised using a rigorous process that is based on the GRADE (Grading of Recommendations Assessment, Development and Evaluation) system of evaluation. ${ }^{9,10}$ With the assistance of our $\mathrm{PhD}$ methodologist (IG), we used the recently developed Canadian CAN-IMPLEMENT ${ }^{\odot}$ process adapted from the ADAPTE Collaboration. ${ }^{11-13}$ We created an Advisory Committee consisting of ten academic emergency physicians (one also expert in thrombosis medicine), four community emergency physicians, three cardiologists, one $\mathrm{PhD}$ methodologist, and two patients. Our focus was four key elements of ED care: assessment and risk stratification, rhythm and rate control, short-term and long-term stroke prevention, and disposition and follow-up. The Advisory Committee communicated by a two-day faceto-face meeting in March 2017, teleconferences,

From the *Department of Emergency Medicine, University of Ottawa, Ottawa, ON; †Ottawa Hospital Research Institute, Clinical Epidemiology Program, Ottawa, ON; ‡Department of Emergency Medicine, University of British Columbia, Vancouver, BC; §Department of Family Medicine and Emergency Medicine, Université de Montréal, Montreal, QC; IEmergency Medicine Services, Montreal Heart Institute, Montreal, Canada; IDivision of Cardiology, Terrence Donnelly Heart Centre, St Michael's Hospital, University of Toronto, Toronto, ON; **School of Epidemiology and Public Health, University of Ottawa, Ottawa, ON; †tInstitute for Clinical Evaluative Sciences, Toronto, ON; ¥¥Sunnybrook Health Sciences Centre, Toronto, ON; §§Department of Family Medicine and Emergency Medicine, Université Laval, Laval, OC; TףPeterborough Regional Health Centre, Peterborough, ON; IIIDivision of Emergency Medicine, Department of Medicine, McMaster University, Hamilton, ON; ***Department of Emergency Medicine, University of Calgary, Calgary, AB; $\uparrow+\uparrow H e a r t$ Rhythm Services, Division of Cardiology, University of British Columbia, Vancouver,

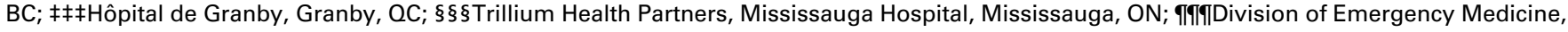
Department of Family Medicine, McMaster University, Hamilton, ON; IIIISt. Mary's General Hospital, Kitchener, ON; ****Ouebec City, OC; ††††Ottawa, ON; and the ¥¥¥‡Division of Cardiology, Western University, London, ON.

Correspondence to: Dr. Ian G. Stiell, Clinical Epidemiology Unit, F657, The Ottawa Hospital, 1053 Carling Avenue, Ottawa, ON K1Y 4E9 Canada; Email: istiell@ohri.ca 
and email. The checklist was prepared and revised through a process of feedback and discussions on all issues by all panel members. These revisions went through ten iterations until consensus was achieved. We then circulated the draft checklist for comment to approximately 300 emergency medicine and cardiology colleagues; their email written feedback was further incorporated and the final version created and approved by the panel.

During the consensus and feedback processes, we addressed a number of issues and concerns, some of which required extensive discussion. We spent considerable time defining what is meant by "unstable" and highlighting the issue that many unstable patients are actually suffering from underlying medical problems rather than a primary arrhythmia. Where possible we chose to simplify the checklist, for example listing only procainamide for pharmacological cardioversion. Other drugs were considered including vernakalant, ibutilide, propafenone, flecainide, and amiodarone. We also tried to give specific drug dosage recommendations, recognizing that physicians are free to consult any number of excellent pharmaceutical references. The panel believes that, overall, a strategy of ED cardioversion and discharge home from the ED is preferable from both the patient and the healthcare system perspective, for most patients. One controversial recommendation is to consider rate control or transesophageal echocardiography (TEE)-guided $\mathrm{CV}$ if the duration of symptoms is 24-48 hours and the patient has two or more CHADS-65 criteria. This is based on some recent data from Finland. ${ }^{14,15}$ We emphasize the importance of evaluating long-term stroke risk by use of the CHADS-65 algorithm and encourage ED physicians to prescribe anticoagulants where indicated.

Our hope is that the CAEP Acute Atrial Fibrillation/ Flutter Best Practices Checklist will standardize and improve care of AAF and AAFL in large and small EDs alike. We believe that these patients can be managed rapidly and safely, with early ED discharge and return to normal activities.

\section{SUPPLEMENTARY MATERIAL}

To view supplementary material for this article, please visit https://doi.org/10.1017/cem.2018.26

Acknowledgments: Funding for this guideline was supported by the Cardiac Arrhythmia Network of Canada (CANet) as part of the Networks of Centres of Excellence (NCE). IS has received funding from Boehringer Ingelheim Canada Ltd. and from JDP Therapeutics for participation in clinical studies. PA has received research funding and/or honoraria from BMS-Pfizer Alliance, Boehringer Ingelheim, and Servier, KD has received research funding from Bayer. MD has received honoraria and research funding from Biosense Webster, Bayer, Bristol-Myers-Squibb, Abbott, and Servier. AS has received honoraria from Boehringer Ingelheim, Bayer, Pfizer, and Servier. TT has received honoraria from Cardiome Pharma Corp. We thank the hundreds of Canadian emergency physicians and cardiologists who reviewed the draft guidelines and who provided very helpful feedback.

\section{REFERENCES}

1. Connors S, Dorian P. Management of supraventricular tachycardia in the emergency department. Can 7 Cardiol 1997;13(Suppl A):19A-24A.

2. Michael JA, Stiell IG, Agarwal S, Mandavia DP. Cardioversion of paroxysmal atrial fibrillation in the emergency department. Ann Emerg Med 1999;33:379-87.

3. Scheuermeyer FX, Innes G, Pourvali R, et al. Missed opportunities for appropriate anticoagulation among emergency department patients with uncomplicated atrial fibrillation or flutter. Ann Emerg Med 2013;62(6):557-65.

4. Atzema CL, Yu B, Ivers N, et al. Incident atrial fibrillation in the emergency department in Ontario: a populationbased retrospective cohort study of follow-up care. CMAJ Open 2015;3(2):E182-91.

5. Stiell IG, Clement CM, Rowe BH, et al. Outcomes for ED Patients with Recent-onset Atrial Fibrillation and Flutter (RAFF) Treated in Canadian Hospitals. Ann Emerg Med 2017;69(5):562-71.

6. Stiell IG, Macle L. Canadian cardiovascular society atrial fibrillation guidelines 2010: management of recent-onset atrial fibrillation and flutter in the emergency department. Can 7 Cardiol 2011;27(1):38-46.

7. Verma A, Cairns JA, Mitchell LB, et al. Focused update of the canadian cardiovascular society guidelines for the management of atrial fibrillation. Can 7 Cardiol 2014;30(10): 1114-30.

8. Macle L, Cairns J, Leblanc K, et al. Focused Update of the Canadian Cardiovascular Society Guidelines for the Management of Atrial Fibrillation. Can 7 Cardiol 2016;32(10): 1170-85.

9. Gillis AM, Skanes AC. Canadian Cardiovascular Society atrial fibrillation guidelines 2010: implementing GRADE and achieving consensus. Can 7 Cardiol 2011;27(1): 27-30.

10. Guyatt GH, Oxman AD, Vist GE, et al. GRADE: an emerging consensus on rating quality of evidence and strength of recommendations. BMF 2008;336(7650):924-6.

11. The ADAPTE Collaboration. Guideline Adaptation: A Resource Toolkit. Version 2.0; 2009. Available at: http:// www.g-i-n.net/document-store/working-groups-documents/ adaptation/adapte-resource-toolkit-guideline-adaptation-2-0. pdf. 
12. Harrison MB, Graham ID, van den Hoek J, et al. Guideline adaptation and implementation planning: a prospective observational study. Implement Sci 2013;8:49.

13. Harrison MB, van den Hoek J, Graham ID. $C A N$ IMPLEMENT: Planning for best-practice implementation. Philadelphia: Lippincott Williams \& Wilkins; 2014.
14. Nuotio I, Hartikainen JE, Gronberg T, et al. Time to cardioversion for acute atrial fibrillation and thromboembolic complications. FAMA 2014;312(6):647-9.

15. Stiell IG, Healey JS, Cairns JA. Safety of urgent cardioversion for patients with recent-onset atrial fibrillation and flutter. Can 7 Cardiol 2015;31(3):239-41.

\section{APPENDICES}

CAEP Acute Atrial Fibrillation/Flutter Best Practices Checklist

Panelists

\begin{tabular}{l} 
Academic HSC Emergency Medicine \\
\hline lan Stiell - Ottawa \\
Frank Scheuermeyer - UBC \\
Suneel Upadhye - McMaster \\
Clare Atzema - Toronto \\
Andrew McRae - Calgary \\
Kerstin de Wit - McMaster (Thrombosis) \\
Patrick Archambault - Laval \\
Warren Cheung - Ottawa \\
Debra Eagles - Ottawa \\
Alain Vadeboncoeur - Montreal \\
Methodologist \\
lan Graham - Ottawa \\
Ottawa Staff \\
Cathy Clement \\
Jennifer Brinkhurst
\end{tabular}

Community Hospital Emergency Medicine

Troy Tebbenham - Peterborough

Rick Mann - Mississauga

Rupinder Sahsi - Kitchener

Geneviève Baril - Granby

Cardiology

Allan Skanes - Western

Paul Angaran - Toronto

Marc W. Deyell - UBC

Patients

Christian Chabot - Quebec City

David Gibbons - Ottawa 


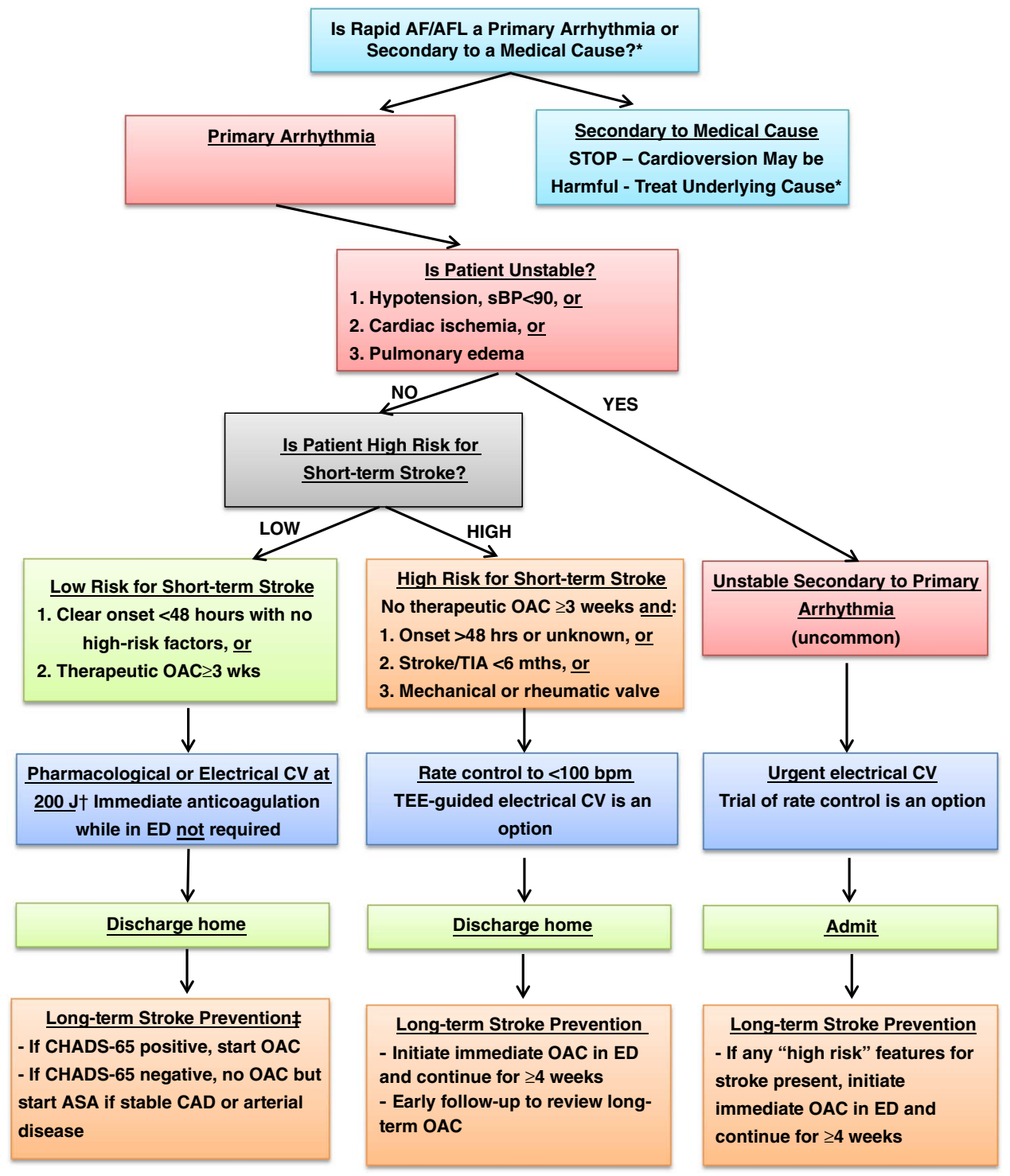

Figure 1. Overall management algorithm for patients presenting to the ED with acute atrial fibrillation or flutter. Adapted from CCS 2014 Figure 2. ${ }^{7}$

Notes.

* Consider medical cause (e.g. sepsis, bleeding, PE, heart failure, ACS, etc) if not sudden onset, HR $<150$, fever, known permanent AF; cardioversion may be harmful, rate control discouraged; investigate and treat underlying condition aggressively

† Consider rate control or transesophageal echocardiography (TEE)-guided CV if duration 24-48 hrs and two or more CHADS-65 criteria

₹ If CHADS-65 positive, start OAC; if stable CAD, discontinue ASA; if CAD with other anti-platelets or recent PCl, consult cardiology (see Figure 2)

ASA = acetylsalicylic acid; CAD = coronary artery disease; CHADS-65 = age 65, congestive heart failure, hypertension, age diabetes, stroke / transient ischemic attack; $\mathrm{CV}=$ cardioversion; $\mathrm{NOAC}=$ novel direct oral anticoagulant; $\mathrm{OAC}=\mathrm{oral}$ anticoagulant; $\mathrm{TI} A=$ transient ischemic attack. 


\section{$\underline{\text { A. Assessment and Risk Stratification }}$}

1a) Is Rapid AF/AFL a Primary Arrhythmia or Secondary to Medical Causes?

$\square$ Secondary to medical causes (usually in patients with pre-existing/permanent AF), e.g. sepsis, bleeding, $P E$, heart failure, ACS, etc

- Investigate and treat underlying causes aggressively

- Cardioversion may be harmful

Primary arrhythmia

- Avoid rate control

TIP: More likely to be secondary to medical cause if:

- Not sudden onset, no palpitations

- Known permanent AF, on OACs, old ECGs show AF

- No history of ED cardioversions

$-H R<150$

- Fever, dyspnea, pain

\section{1b) Is the Patient Unstable?}

- Unstable due to acute primary AF/AFL is uncommon, except for AF with rapid ventricular pre-excitation (WPW)

$\square$ i) Hypotension: $\mathrm{s} B P<90$, or signs of shock (e.g. altered mental status)

$\square$ ii) Cardiac ischemia: ongoing severe chest pain or marked ST depression ( $>2 \mathrm{~mm}$ ) on ECG despite therapy

iii) Pulmonary edema: significant dyspnea, crackles, and hypoxia

\section{2a) Stable Low-Risk for Short-term Stroke}

Clear onset $<\mathbf{4 8}$ hours, $\underline{\text { OR }}$

- If 24-48 hrs and two or more CHADS-65 criteria, may not be low risk

NOAC or therapeutic warfarin for at least 3 weeks

\section{2b) Stable High-Risk for Short-term Stroke}

\section{No/Inadequate OAC, $\underline{\text { AND }}$}

$\square$ One of:

$\square$ Onset $>48$ hours or unknown, $\underline{\text { or }}$

$\square$ Stroke/TIA $<\mathbf{6}$ months or

$\square$ Valvular heart disease: Mechanical (INR should be $>2.5$ ) or rheumatic mitral stenosis

TIP: How to determine if therapeutic OAC $x 3$ weeks?

- Based on MD judgment

- NOAC - confirm compliance by history

- Warfarin

○ Current INR $>2.0$ ?

- Recent INR values $>2.0$ ?

- Recent INR testing confirmed by history?

- No recent changes in dose? 


\section{B. Rhythm and Rate Control}

\section{1) Unstable due to Primay Arrhythmia}

- Unstable due to acute AF/AFL is very uncommon

$\square$ Urgent electrical CV if onset $<48$ hrs or WPW

$\square$ Consider trial of rate control if onset $>48 \mathrm{hrs}$

\section{2a) Stable Low-Risk for Short-term Stroke}

Rhythm control preferable, patient quality of life, shorter length of stay, fewer hospital resources

- Immediate anticoagulation in ED not required

- If onset 24-48 hrs and two or more CHADS-65 criteria, consider rate control or TEE-guided CV

Rate control acceptable, per patient and physician preference

- E.g. elderly patients who are minimally symptomatic

\section{2b) Stable High-Risk for Short-term Stroke}

Rate control recommended

Rhythm control only if cleared by transesophageal echocardiography (TEE)

- Requires bridging with LMW heparin or NOAC

\section{3a) Rhythm Control}

- Either pharmacological or electrical cardioversion acceptable, per patient and physician preference

- consider previous episodes; if one doesn't work, try the other

- Pre-treatment with rate control agents not recommended - ineffective and delays treatment

$\square$ Pharmacological cardioversion

$\square$ Procainamide IV - $15 \mathrm{mg} / \mathrm{kg}$ in $500 \mathrm{ml}$ NS over 30-60 minutes

- avoid if $S B P<100 \mathrm{~mm} \mathrm{Hg}$ or QTc $>500 \mathrm{msec}$

- interrupt infusion if BP drops or QRS lengthens visibly (i.e. $>30 \%$ )

- check QTc after conversion

- Amiodarone IV not recommended - slow, low efficacy

- Less commonly used options include: vernakalant IV, ibutilide IV, propafenone PO, and flecainide $\mathrm{PO}$

Electrical cardioversion

$\square$ Setup - minimum 2 staff (RN/RRT; RN/RN), $2^{\text {nd }}$ physician ideal

$\square$ Procedural sedation per local practice - e.g. Fentanyl, Propofol

$\square$ Pad/paddle position - either antero-lateral or antero-posterior acceptable

- avoid sternum, breast tissue; if failure, apply pressure with paddles, try the other position

Start with 200 joules synchronized - avoid starting with low energy level

- Most patients can be discharged within 30 minutes of conversion

\section{3b) Rate Control}

- Calcium channel- and beta- blockers considered first line

- If patient already taking oral calcium-channel or beta-blocker, choose same drug group first

- If difficulty achieving adequate rate control, consider using the other first-line agent, IV digoxin, or cardiology consultation

- $\quad \square$ Calcium channel blocker: avoid if acute heart failure or known LV dysfunction

- Diltiazem $0.25 \mathrm{mg} / \mathrm{kg}$ IV over 10 minutes; repeat q15-20 min at $0.35 \mathrm{mg} / \mathrm{kg}$ up to 3 doses 
- Start 30-60 mg PO within 30 mins of effective IV rate control

- Discharge on 30-60mg QID or Extended Release 120-240 mg once daily

Beta Blocker - Metoprolol 2.5-5 mg IV over 2 minutes, repeat q15-20 min up to 3 doses

- Start 25-50 mg PO within 30 mins of effective IV rate control

- Discharge on 25-50 mg BID

Digoxin is second line, as slow onset $-0.25-0.5 \mathrm{mg}$ loading dose, then 0.25mg IV q4-6h to a maximum of 1.5 mg over 24 hours; caution in renal failure

- Consider first line if hypotension or acute $H F$

Heart rate target achieved: $<100$ bpm at rest, $<110$ walking

\section{4) Rapid Ventricular Pre-Excitation (WPW)}

$\square$ Urgent electrical CV

$\square$ Procainamide IV if stable

- AV nodal blocking agents contraindicated: digoxin, calcium channel blockers, beta-blockers, adenosine, amiodarone

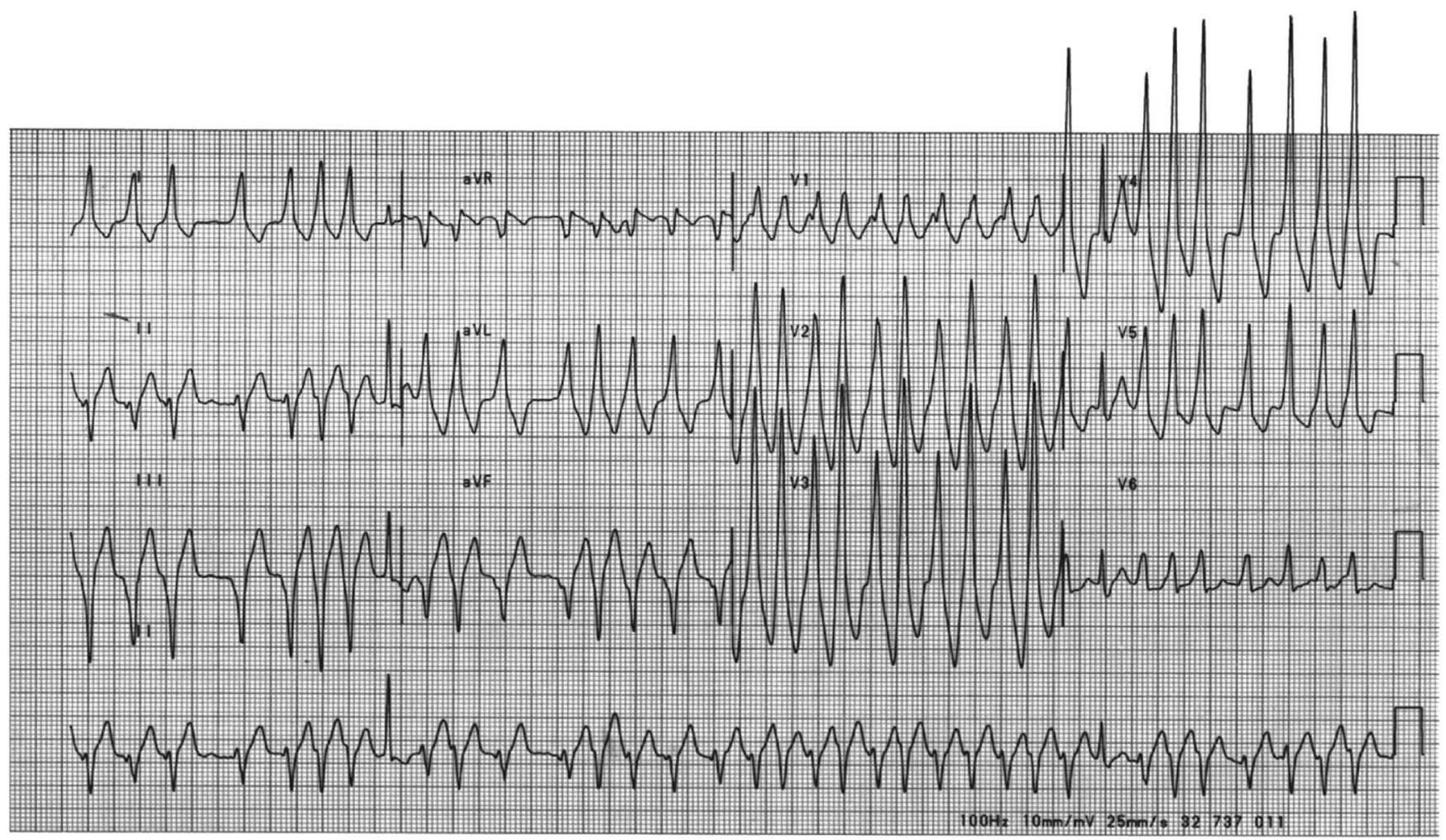

Figure 2. Rapid Ventricular Pre-Excitation 


\section{Long-term Stroke Prevention}

\section{1) CHADS-65 Algorithm ${ }^{8}$-Figure 3}

- Antithrombotic therapy prescribed at discharge is for long-term prevention of strokes

$\square$ If CHADS-65 positive, initiate oral anticoagulation

- NOACs preferred over warfarin

- Use warfarin if mechanical valve, rheumatic mitral stenosis, severe renal impairment $(\mathrm{CrCl}<30$ $\mathrm{ml} / \mathrm{min}$ )

- To decrease bleeding risk: exercise caution if very high fall risk; advise discontinuation of NSAIDs and heavy drinking

- If stable CAD, discontinue ASA

- If CAD with other anti-platelets or recent $\mathrm{PCI}<12$ mos, consult cardiology

If CHADS-65 negative, no oral anticoagulation

$\square$ If CHADS-65 negative and stable coronary, aortic, or peripheral vascular disease, initiate ASA- $81 \mathrm{mg}$ daily

- Patients already taking anti-platelet agents require follow-up with cardiology

If TEE-guided CV, must initiate NOAC immediately $\mathbf{x} 4$ weeks (for rapid onset)

- If warfarin, need LMW heparin bridging

- Need follow-up with cardiology for long-term stroke prevention

- Patients converting spontaneously before ED treatment should generally be prescribed OAC according to the CHADS-65 criteria

- Physicians prescribing OACs should consider shared decision making to include patients preferences with regards to risks and benefits

\section{2a) NOACs}

- See Thrombosis Canada App for details; avoid in pregnancy, breastfeeding

- Do not use if $\mathrm{CrCl}<\mathbf{3 0} \mathrm{ml} / \mathrm{min}$

- Provincial formularies may require Limited Use codes, e.g. failure of warfarin or INR monitoring not possible

$\square$ Dabigatran - $150 \mathrm{mg}$ BID; use $110 \mathrm{mg}$ BID if age $>80$ years, or $>75$ years with bleeding risk

Rivaroxaban - $20 \mathrm{mg}$ daily; use $15 \mathrm{mg}$ daily if $\mathrm{CrCl} 30-49 \mathrm{ml} / \mathrm{min}$

Apixaban $-5 \mathrm{mg}$ BID; use $2.5 \mathrm{mg}$ BID if two of: 1) serum creatinine $\geq 133 \mathrm{umol} / \mathrm{L}, 2)$ age $\geq 80$ years, or 3) body weight $\leq 60 \mathrm{~kg}$

Edoxaban - $60 \mathrm{mg}$ daily; use $30 \mathrm{mg}$ daily if $\mathrm{CrCl} 30-50 \mathrm{ml} / \mathrm{min}$ or weight $\leq 60 \mathrm{~kg}$; important drug interactions

\section{2b) Warfarin}

Initiate warfarin: $5 \mathrm{mg}$ daily; $1-2 \mathrm{mg}$ daily if frail, low weight, Asian descent

- Heparin bridging not required unless TEE-guided CV 


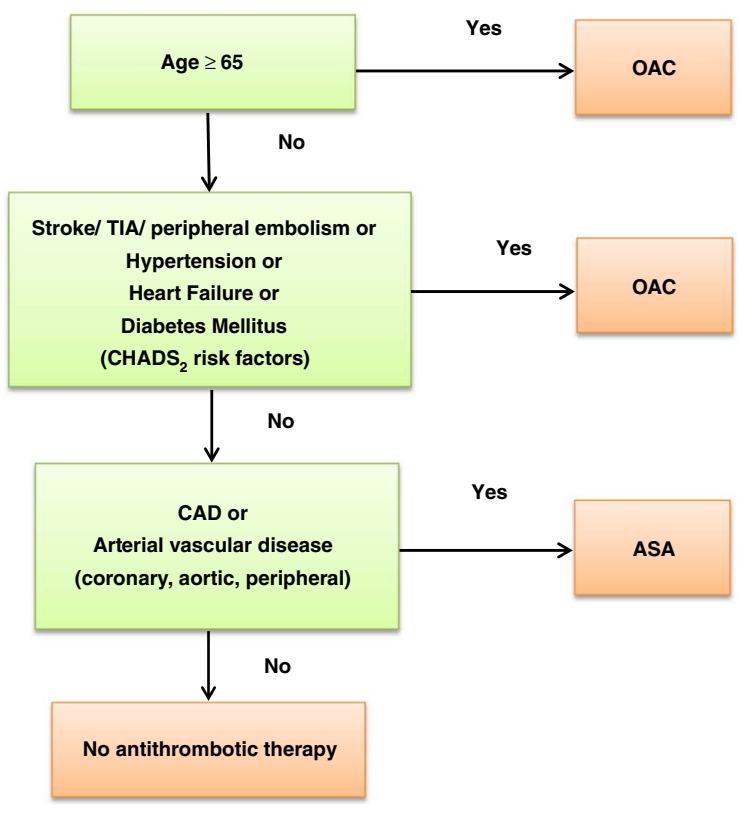

Figure 3. "CCS algorithm" ("CHADS65") for long-term stroke prevention in AF

\section{Disposition and Follow-up}

\section{1) Admission to Hospital}

- Patients rarely require hospital admission for uncomplicated acute AF/AFL unless they:

$\square$ are highly symptomatic despite adequate treatment

$\square$ have ACS with significant chest pain, troponin rise, and ECG changes

- no need to routinely measure troponin, small demand rise expected

have acute heart failure not improved with ED treatment

\section{2) Anti-thrombotic Therapy for Long-term Stroke Prevention}

$\square$ If CHADS-65 positive, prescribe NOAC or warfarin, regardless of whether rhythm or rate control used $\square$ If CHADS-65 negative and stable CAD or arterial vascular disease, continue or prescribe ASA

\section{3) Follow-up Issues}

Ensure INR monitoring at 3-5 days if warfarin initiated

Recommend physician follow-up $<7$ days, if new warfarin or rate control meds

Recommend cardiology / internal medicine follow-up in 4-6 weeks if not already followed or if new medications prescribed

$\checkmark$ Provide handout describing new medication, atrial fibrillation, and follow-up; early renal function monitoring if new NOAC; Thrombosis Canada has patient information sheets

- Do not initiate anti-arrhythmic agents like amiodarone or propafenone in the ED

- If sinus rhythm achieved, generally no need to initiate beta- or calcium channel-blockers 